\title{
Visual Outcomes of Plasma Exchange Treatment of Steroid-Refractory Optic Neuritis: A Retrospective Monocentric Analysis
}

\author{
Nic Skorupka ${ }^{a}$ Andrei Miclea ${ }^{a}$ Katarzyna Aleksandra Jalowiec ${ }^{b}$ \\ Christoph Bocksrucker $^{b}$ Nicole Kamber ${ }^{a} \quad$ Andrew Chan ${ }^{a}$ \\ Behrouz Mansouri Taleghani $^{b}$ Robert Hoepner ${ }^{a} \quad$ Anke Salmen ${ }^{a}$ \\ ${ }^{a}$ Department of Neurology, Inselspital, Bern University Hospital, University of Bern, Bern, Switzerland; ${ }^{b}$ Department \\ of Hematology, Inselspital, Bern University Hospital, University of Bern, Bern, Switzerland
}

\section{Keywords \\ Multiple sclerosis · Relapse · Apheresis · Visual acuity}

\begin{abstract}
Introduction: In acute inflammatory optic neuritis (ON) as a typical onset of multiple sclerosis (MS), only few studies have investigated plasma exchange (PLEX) as a sequential treatment after insufficient response to high-dose intravenous glucocorticosteroids. Therefore, we aimed to investigate treatment outcome on visual acuity (VA) with PLEX in patients with steroid-refractory ON. Methods: In our retrospective monocentric study, medical records were screened for patients with acute $\mathrm{ON}$ as their first relapse with sequential MS diagnosis or with an established MS diagnosis from the Bern University Hospital (Switzerland) that were treated with PLEX between 2016 and 2018 due to lacking steroid response. VA prior to steroid administration, and before and after PLEX were assessed and compared using the Friedman multiple comparison test. Results: In total, 18 patients were included in the analysis. Interval from symptom onset to PLEX was 20.3 days (mean, 95\% Cl 14.8-25.9). Relevant functional improvement (VA of $\geq 0.5$, after a mean of 15.9 (13.318.5) days after start of PLEX) was detected in 16/18 (88.9\%) with a significant amelioration as compared to VA before glucocorticosteroids and before PLEX $(p<0.0001)$. VA improvement at a later time point (38.1 weeks, 25.2-51.0) was present in 15/16 (93.8\%) patients. No serious adverse events
\end{abstract}

were detected. PLEX could be performed via peripheral access in $13 / 18$ patients (72.2\%). Conclusion: Our study demonstrates significant improvements of VA with PLEX in a cohort of MS patients with steroid-refractory ON. High response rates may be due to the timely treatment initiation. Despite the small sample size, our data support the early use of PLEX in steroid-refractory ON with a favorable safety profile.

C 2019 S. Karger AG, Basel

\section{Introduction}

Optic neuritis $(\mathrm{ON})$ describes an acute demyelinating event, which can occur isolated or as the first manifestation of multiple sclerosis (MS) [1-3]. Approximately 50\% of patients with ON eventually develop MS [1]. The clinical picture of a relative afferent pupillary deficit, pain during eye movement, and a normal or mildly edematous optic disc are typical clinical signs of ON [4]. However, the most prominent symptom is the decrease in visual acuity (VA) which accounts for a relevant component of MS-associated permanent disability $[5,6]$.

MRI characteristics and novel diagnostic means such as optical coherence tomography help to better dissect the

N.S., A.M., R.H. and A.S. contributed equally to this work.

\section{KARGER}

(C) 2019 S. Karger AG, Basel 
morphological features of $\mathrm{ON}[6,7]$. Gadolinium enhancement of the optic nerve on the MRI can be found in up to $94 \%$ of patients and may help in diagnosing ON [8]. Yet, due to the lack of standardization and broad application in clinical routine, these diagnostic means are currently not part of the diagnostic criteria for an MS diagnosis [9].

Treatment of ON and other relapses in MS patients is still mainly based on high-dose intravenous (i.v.) glucocorticosteroids [10,11]. Nevertheless, ON outcomes comparing placebo and i.v. glucocorticosteroids are contradictory [12-14]. In the first prospective ON study, visual function recovered faster in the patient group receiving i.v. methylprednisolone versus placebo. However, at 6 months, the group that had received i.v. methylprednisolone had only slightly better visual fields, contrast sensitivity and color vision, but no better VA [13]. This study further assessed whether new ON episodes had occurred in between (and whether the risk of a new episode differs between groups); however, it did not describe whether the new episode is the major contributor to a visual deficit during follow-up. A Cochrane review concludes that there is no evidence of beneficial long-term effects from glucocorticosteroids compared to placebo for VA, visual field and contrast sensitivity outcomes [14]. Yet, considering a relapsing disease with a high likelihood of a sequential relapse in the same affected domain [15], this is not a surprising result.

In case of lacking efficacy, sequential high-dose i.v. glucocorticosteroids and plasma exchange (PLEX) or immunoadsorption (IA) may be considered [10, 11]. Evidence on PLEX and IA in MS relapses is based on observational prospective and retrospective data showing overall beneficial effects [16-21], even in special situations such as a severe relapse during pregnancy [22]. Yet, these studies describe steroid-refractory relapses in general, not dissecting for specific relapse symptoms or even with different underlying conditions (MS and neuromyelitis optica spectrum disorders [NMOSD]), and are too small to form subgroups by symptomatic presentation. Moreover, disease heterogeneity seems to account for relevant differences in response to PLEX as demonstrated histopathologically [23].

Only 4 studies were identified to specifically assess PLEX [24, 25], IA [26] or a mixed PLEX/IA setup [27] in ON of different etiology with $n=34, n=23, n=11$ and $n=48$ patients, respectively. Whereas no influence of PLEX/IA on VA was detected in the study by Faissner et al. [27], with some positive changes in electrophysiological findings, the 3 other studies report significant functional improvements in 56-73\% [24-26]. In a study with 21 relapses in 20 patients, a response for the ON subgroup $(n=12)$ is given with $76 \%$ [20]. This basis of evidence is still poor.

We therefore aimed to investigate visual outcome in a retrospective analysis of patients with $\mathrm{ON}$ and a newly diagnosed or established MS.

\section{Methods}

Study Design, Participants, and Ethics Approval

For our retrospective study, medical records were screened for patients with $\mathrm{ON}$ as the first relapse with consecutive MS diagnosis or with an established diagnosis of MS from the Bern University Hospital (Bern, Switzerland) who were treated with PLEX between 2016 and 2018 due to unresponsiveness to prior application of high-dose i.v. glucocorticosteroids.

We defined unresponsiveness to high-dose i.v. glucocorticosteroids as given per indication for PLEX in clinical routine. The general treatment approach in our center follows the German guidelines on treatment of steroid-refractory MS relapses [11]: PLEX is usually performed after one to two steroid treatment cycles with five sessions of PLEX and an individual decision on further PLEX sessions after the fifth respecting contraindications.

All patients with the necessary documented data were included $(n=18 ; 3$ patients were excluded due to lacking information on initial VA). No further inclusion or exclusion criteria were set.

All data were acquired during routine clinical practice. Besides basic patient and demographic characteristics, total dosage of i.v. glucocorticosteroids, total number of PLEX sessions and the respective time intervals from symptom onset as well as PLEX setting (inpatient vs. outpatient, central vs. peripheral access), and adverse events were assessed (Table 1).

VA was assessed in clinical routine during the standardized neurological examination as part of the Expanded Disability Status Scale (EDSS) [28] by trained physicians using a Snellen chart at $5 \mathrm{~m}$ distance with the patient's usual refraction correction if needed. It is given in decimal numbers. VA was evaluated at the following time points: before i.v. glucocorticosteroid treatment (mean 8.8 days, $95 \%$ confidence interval [95\% CI] 4.4-13.2, after onset, $n=18$ ), after i.v. glucocorticosteroid therapy, i.e. before PLEX (19.9 [14.1-25.8] days after onset, $n=18$ ), and after PLEX (early: 36.2 [29.8-42.6] days after onset, $n=18$, and late: 38.1 [25.2-51.0] weeks after onset, $n=16$ ).

The responsible cantonal ethics committee approved this study and waived separate informed consent as for the inclusion period, the general consent was valid (registration No. KEK-BE 201701369). General consent status was checked for all patients prior to any analysis.

\section{PLEX Procedure}

PLEX was performed using Spectra Optia (Terumo BCT $\left.{ }^{(}\right)$. Blood flow was aimed to reach $50-70 \mathrm{~mL} / \mathrm{min}$, with variations depending on the venous access and hematocrit of the patient. For extracorporeal anticoagulation, we used acid-citrate-dextrose formula $A$ at a rate of 1:12-1:16. Removed plasma was substituted starting with sodium chloride $0.9 \%$ followed by albumin $5 \%$ with fluid balance of $110 \%$. For the first exchange of a series, the volume of both solutions was equal. For the second and all following PLEX of a series, we switched to albumin $5 \%$ after having processed $1 / 3$ of the scheduled plasma volume.

This algorithm is the standard of care in our setting and has been in use, also for therapeutic PLEX in MS, for more than 20 years. Although the current ASFA guidelines (2019) recommend albumin 5\% in therapeutic PLEX [29], there is still little evidence on the best practice for replacement fluids [30]. Therefore, the established algorithm has thus far not been adapted.

Fibrinogen was measured once, directly before every PLEX session. We routinely administered OctaplasLG ${ }^{\circledR} 200-400 \mathrm{~mL}$ (Octapharma AG, Switzerland) at the end of a PLEX session in case of fibrinogen serum levels below $1.0 \mathrm{~g} / \mathrm{L}$. 
Table 1. Demographic and clinical data of the cohort

\begin{tabular}{|c|c|c|}
\hline & $n(\%)$ & Mean $(95 \% \mathrm{CI})$ \\
\hline Female sex & $13 / 18(72.2 \%)$ & \\
\hline Age, years & & $35.4(29.9-40.9)$ \\
\hline Steroid dose, mg & & $6,555.6(4,797.6-8,313.5)$ \\
\hline \multicolumn{3}{|l|}{ Affected eye } \\
\hline Left & $13 / 18(72.2 \%)$ & \\
\hline Right & $4 / 18(22.2 \%)$ & \\
\hline Bilateral & $1 / 18(5.6 \%)$ & \\
\hline 1st event and MS diagnosis & $13 / 18(72.2 \%)$ & \\
\hline \multicolumn{3}{|l|}{ Immunotherapy } \\
\hline No & $15 / 18(83.3 \%)$ & \\
\hline Glatiramer acetate & $1 / 18(5.6 \%)$ & \\
\hline Interferon beta formulations & $2 / 18(11.1 \%)$ & \\
\hline \multicolumn{3}{|l|}{ Time intervals } \\
\hline Symptom onset to 1 st steroid, days & & $8.4(4.2-12.5)$ \\
\hline Symptom onset to PLEX, days & & $20.3(14.8-25.9)$ \\
\hline Number of PLEX sessions (available for $n=17$ ) & & $6.5(6.0-7.0)$ \\
\hline Setting of PLEX (fully ambulatory setting) & $10 / 18(55.6 \%)$ & \\
\hline PLEX aditus (fully via peripheral veins) & $13 / 18(72.2 \%)$ & \\
\hline \multicolumn{3}{|l|}{ Adverse events } \\
\hline Any & $13 / 18(72.2 \%)$ & \\
\hline Dizziness & $2 / 18(11.1 \%)$ & \\
\hline Hypocalcemic symptoms & $1 / 18(5.6 \%)$ & \\
\hline Hypotonia & $2 / 18(11.1 \%)$ & \\
\hline Low fibrinogen & $11 / 18(61.1 \%)$ & \\
\hline Muscular complaints & $1 / 18(5.6 \%)$ & \\
\hline Nausea & $2 / 18(11.1 \%)$ & \\
\hline
\end{tabular}

The standard plasma volume exchanged per session was 1.2 times of the patient's plasma volume, and deviations from this setting only occurred when the peripheral access was not sufficient for the whole PLEX session ( $n=2$ sessions in 2 patients, see below).

Patients were treated on consecutive days or every other day with a 2-day break over weekends, mainly depending on infrastructural circumstances. Only $3 / 18$ patients did not receive any session on consecutive days.

\section{Statistical Analysis}

For categorical variables, absolute and relative frequencies are reported. Continuous variables are expressed as mean with $95 \%$ CI. VA before i.v. glucocorticosteroids and before and early after PLEX were compared using the Friedman multiple comparison test. If both eyes were affected by $\mathrm{ON}(n=1)$, the eye with the worse VA was included in the analysis. Contingency analyses were performed using Fisher's exact test. A $p$ value $<0.05$ was defined to demonstrate significant findings.

\section{Results}

\section{Cohort}

We identified 18 patients with sufficient data (documented VA at least at the time points prior first glucocorticosteroid administration, prior initiation of PLEX and early after PLEX). The basic demographic and clinical data are summarized in Table 1.

In our cohort, 13 of 18 patients were female $(72.2 \%)$ with a mean age at onset of ON of 35.4 (95\% CI 29.940.9). For the same proportion of patients, ON represented the first event with the sequential diagnosis of relapsing-remitting MS. Thus, the majority of patients was untreated (83.3\%). ON was mostly unilateral, except for 1 patient in whom exclusion of causes other than MS was extensively performed without significant findings (NMOSD, anti-MOG antibodies, sarcoidosis, vasculitis, infectious disorders).

The mean glucocorticosteroid dosage reflects highdose treatment with two sequential cycles in most patients. The time interval until treatment initiation was short (8.4 [4.2-12.5] days).

\section{VA prior to PLEX}

Individual visual outcome is depicted in Figure 1. Mean VA before glucocorticosteroid administration was 0.24 (0.15-0.34), reflecting severe visual impairment. In all patients with initial VA of 0.50 or higher, further deterioration of VA occurred despite glucocorticosteroid 
Fig. 1. Course of visual acuity of the affected eye $(n=18)$. Visual acuity prior to glucocorticosteroid treatment (GC), prior to initiation of plasma exchange (PLEX), i.e. after GC treatment, and after PLEX. Overlapping values of individual patients are not represented separately, single lines may thus represent more than 1 patient. For $n=$ 1 patient with bilateral optic neuritis, the worse eye was included in the analysis. Matched observations were tested with the Friedman multiple comparison test. n.s., not significant; $p$ value for significant findings $<0.0001$.

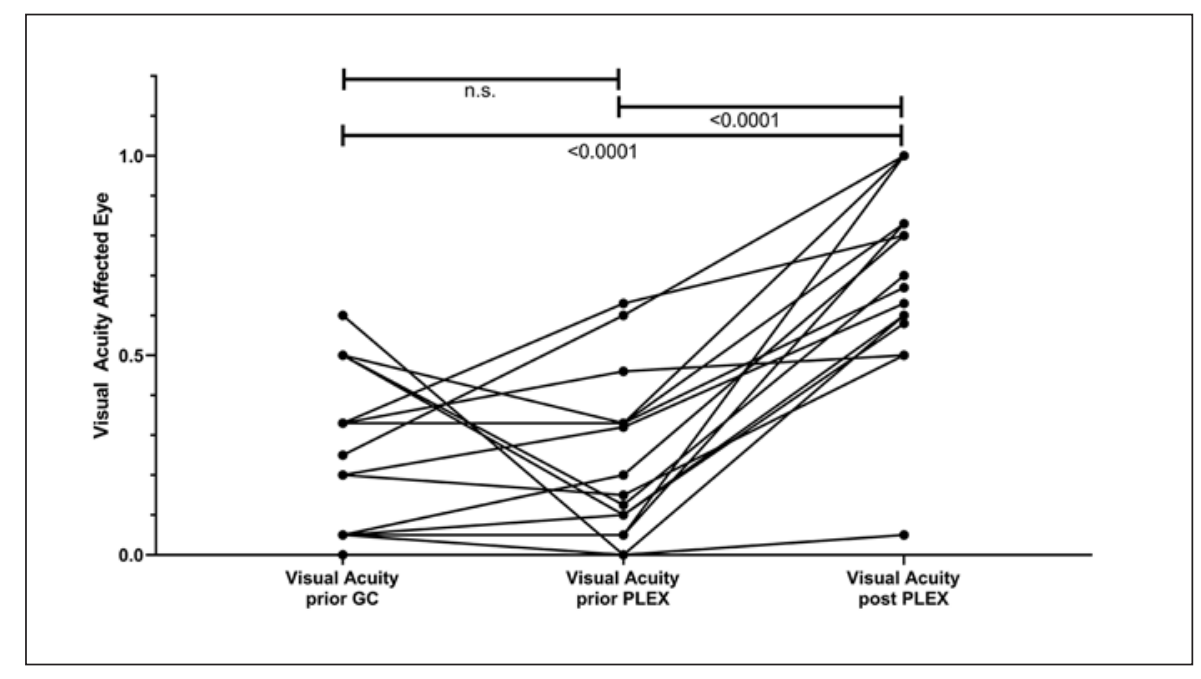

treatment (Fig. 1). For 2 patients, VA was 0.60 and 0.63 , respectively, before initiation of PLEX, reflecting partial glucocorticosteroid response, and resulting in a mean $\mathrm{VA}$ before PLEX of the cohort of 0.22 (0.12-0.32).

\section{Efficacy}

Time interval from symptom onset to initiation of PLEX was short (20.3 [14.8-25.9] days, Table 1).

Relevant functional improvement (VA of $\geq 0.5$, documented after a mean of 15.9 [13.3-18.5] days after start of PLEX) was detected in 16/18 (88.9\%). In 2 patients, unilateral functional amaurosis (VA 0.0-0.05) could not be modified with glucocorticosteroids or PLEX (Fig. 1). Visual outcome as measured by VA was significantly improved via PLEX as compared to VA before glucocorticosteroids and before PLEX (Friedman multiple comparison test, $p<0.0001$, Fig. 1$)$.

For 16 patients, VA of the affected eye was available at later time points after initial onset of ON (mean 38.1 weeks [25.2-51.0]). Here, 15/16 (93.8\%) patients demonstrated relevant functional improvement (mean VA 0.82 [0.69-0.96]). Interestingly, this includes 1 patient with initial unilateral functional amaurosis early after PLEX (documented at day 13), but relevant improvement to a VA of 0.75 after 20.6 weeks. The other patient with an amaurotic eye remained unchanged after 40.6 weeks.

\section{Safety and Procedural Aspects}

Table 1 lists setting of PLEX and documented adverse events. Patients received 5 to max. 8 sessions of PLEX. In $13 / 18$ patients, PLEX could be performed fully via peripheral venous access (72.2\%); 10/18 patients could be treated in an ambulatory setting (55.6\%).

One patient switched from an outpatient to inpatient setting as the 2 nd cycle with peripheral venous access failed and a central catheter had to be installed. One additional patient (hospitalized from the beginning) switched from a peripheral to central catheter during the procedure.

Commonly described side effects, like dizziness, hypotonia, and hypocalcemic symptoms, were within the usual bounds in our cohort (Table 1). Low fibrinogen was noted in $11 / 18$ patients (61.1\%). Comparing patients treated on consecutive days or not and with or without low fibrinogen, we did not detect significant differences (Fisher's exact test, $p=0.26$ ).

\section{Discussion/Conclusion}

Our retrospective study demonstrates a significant improvement of VA with PLEX in a small cohort of MS patients with steroid-refractory ON. Response rates are high early (88.9\%) and at later time points after PLEX (93.8\%), even in patients with very severe initial visual impairment. The overall safety in our cohort was favorable, with only mild adverse events, hypofibrinogenemia being the most common (61.1\%). The latter patients are regularly substituted in our center, using 200-400 mL OctaplasLG ${ }^{\circledR}$ (Octapharma AG, Switzerland).

Our response rates are considerably higher than previously reported $[24,27]$. The most comparable results with around $70 \%$ with improved vision were described for a different apheresis method (IA, using tryptophanbased columns) [26], in a mixed population of MS, NMOSD, and clinically isolated syndrome [25] or a small subgroup in a case series of 21 relapses [20]. A general explanation may be the small sample size of all cohorts including our own and the mostly retrospective data collection with potential sampling bias [20, 24, 25, 27]. However, in our study, the 3 excluded patients without initial VA documentation did not differ demographical- 
ly or clinically from the rest of the cohort, and the final outcome was favorable also in these patients (VA 0.81.2). One of the studies analyzed patients with $\mathrm{ON}$ of different etiology including only $38.2 \%$ with MS-associated ON [24]. This is of relevance as MS- and NMOSD-associated ON differ not only pathophysiologically and structurally $[31,32]$, but most importantly in terms of outcome $[32,33]$.

However, additional reasons should be discussed. VA as a simple and rough measure of visual function has been criticized as an outcome measure in MS as more sensitive parameters, e.g. low-contrast VA, at best accomplished by additional means such as optical coherence tomography, seem to better reflect actual impairment of the patients $[5,6]$. Yet, it is remarkable that in our study VA as documented in clinical routine served as a robust outcome parameter.

Time intervals, most importantly symptom onset to PLEX start, in our study were shorter than in those previous studies where it has been distinctly reported [20,24, 26]. As time from onset has been discussed as a potential modifier of treatment response, both in MS [34] and NMOSD relapses [35], this might additionally explain the differences between our results and those in the previous ON cohorts.

Although preliminary, our data argue for rapid and consequent treatment of $\mathrm{ON}$ with severe visual impairment to alleviate persisting functional recovery.

Safety and tolerability of PLEX has overall been described as favorable, as in our study [16, 20, 25, 27]. The high rate of hypofibrinogenemia (61.1\%) may be protocol-associated in our center. However, maybe due to the small sample size, we did not detect significant differences whether patients were treated on consecutive days or not. Treatment of hypofibrinogenemia in our center is performed via substitution with pathogen-inactivated plasma, as this is the standard of care in collaboration with our hematological department. As this is costly, it may well be discussed to suspend the next PLEX session for 1 or more days [20,25], also for economic reasons.

Whereas in some centers, insertion of a central venous catheter is standard of care $[25,27]$, we report a proportion of $72.2 \%$ of patients undergoing the full PLEX procedure via peripheral venous access. This proportion is higher than reported by Trebst et al. [20]. Especially for our patient cohort of mostly newly diagnosed MS patients without preexisting disability, this represents a considerable advantage for patients. This is not only due to the fact that central catheters might be linked to more and potentially severe complications (infections, thrombosis, dislocation, pneumothorax, and others), but also the fact that patients have a chance to avoid hospitalization (55.6\% in our cohort) which might confront them also emotionally more intensively with the disease process.
As we only screened for patients failing glucocorticosteroid treatment, we cannot draw conclusions on the proportion of steroid-refractory $\mathrm{ON}$ in an MS population from our study.

Despite the small sample size, we here present relevant data supporting the early use of PLEX in steroid-refractory ON patients with MS with high recovery rates and favorable safety.

\section{Statement of Ethics}

For this retrospective study, ethics approval was obtained from the responsible local committee (registration No. KEK-BE 201701369). The study was performed in accordance with Swiss law (Humanforschungsgesetz) and with the World Medical Association Declaration of Helsinki. The local ethics committee waived separate informed consent as with the inclusion period of this study, the general consent (GC) procedure was implemented and GC approval status was checked for all subjects prior to any analysis.

\section{Disclosure Statement}

A. Miclea, N. Skorupka, C. Bocksrucker, B. Mansouri Taleghani report no disclosures. K.A. Jalowiec received travel grants from Amgen, Pfizer, Novartis, not related to this work. N. Kamber received travel grants from Biogen, Merck, Genzyme, and Roche and speaker honoraria from Biogen and Roche, not related to this work. A. Chan has received personal compensation for activities with Bayer, Biogen, Genzyme, Merck, Novartis, Roche, Teva. He received research support from the Swiss National Fonds (SNF, No. 310030_172952), Genzyme, and UCB. He serves in the editorial board for Clinical and Translational Neuroscience and the Journal of International Medical Research. R. Hoepner received research and travel grants from Novartis and Biogen Idec. He also received speaker honoraria from Biogen, Novartis, Merck, and Almirall. He is supported by the Swiss Multiple Sclerosis Society. A. Salmen received speaker honoraria and/or travel compensation for activities with Almirall Hermal GmbH, Biogen, Merck, Novartis, Roche, and Sanofi Genzyme, none related to this work.

\section{Funding Sources}

No specific funding for this study.

\section{Author Contributions}

N. Skorupka, A. Miclea collected the data and contributed to the design of the study, analysis, interpretation of the data, and the writing and revision of the manuscript. K.A. Jalowiec, C. Bocksrucker collected the data and contributed to the design of the study, and revision of the manuscript. N. Kamber, A. Chan contributed to the interpretation of the data, and revision of the manuscript. B. Mansouri Taleghani contributed to the design of the study, interpretation of the data, and the writing and revision of the manuscript. R. Hoepner and A. Salmen contributed to the design of the study, analysis, interpretation of the data, and the writing and revision of the manuscript. 


\section{References}

1 Optic Neuritis Study Group. Multiple sclerosis risk after optic neuritis: final optic neuritis treatment trial follow-up. Arch Neurol. 2008 Jun;65(6):727-32.

2 Toosy AT, Mason DF, Miller DH. Optic neuritis. Lancet Neurol. 2014 Jan;13(1):83-99.

3 von Bismarck O, Dankowski T, Ambrosius B, Hessler N, Antony G, Ziegler A, et al. Treatment choices and neuropsychological symptoms of a large cohort of early MS. Neurol Neuroimmunol Neuroinflamm. 2018 Mar; 5(3):e446.

4 Wilhelm H, Schabet M. The Diagnosis and Treatment of Optic Neuritis. Dtsch Arztebl Int. 2015 Sep;112(37):616-25.

5 Nolan RC, Akhand O, Rizzo JR, Galetta SL, Balcer LJ. Evolution of Visual Outcomes in Clinical Trials for Multiple Sclerosis DiseaseModifying Therapies. J Neuroophthalmol. 2018 Jun;38(2):202-9.

6 Soelberg K, Specovius S, Zimmermann HG, Grauslund J, Mehlsen JJ, Olesen C, et al. Optical coherence tomography in acute optic neuritis: A population-based study. Acta Neurol Scand. 2018 Dec;138(6):566-73.

7 Soelberg K, Skejoe HP, Grauslund J, Smith TJ, Lillevang ST, Jarius S, et al. Magnetic resonance imaging findings at the first episode of acute optic neuritis. Mult Scler Relat Disord. 2018 Feb;20:30-6.

8 Kupersmith MJ, Alban T, Zeiffer B, Lefton D. Contrast-enhanced MRI in acute optic neuritis: relationship to visual performance. Brain. 2002 Apr;125(Pt 4):812-22.

9 Thompson AJ, Banwell BL, Barkhof F, Carroll WM, Coetzee T, Comi G, et al. Diagnosis of multiple sclerosis: 2017 revisions of the McDonald criteria. Lancet Neurol. 2018 Feb; 17(2):162-73.

10 Repovic P. Management of Multiple Sclerosis Relapses. Continuum (Minneap Minn). 2019 Jun;25(3):655-69.

11 Gold R, Hanschke S, Hemmer B, Wiendl H. DGN/KKNMS Leitlinie zur Diagnose und Therapie der MS - Online-Version, Stand: 13.08.2014. 2014 [accessed 2019 Sept 7]. Available from: http://wwwkompetenznetz-multiplesklerosede/fachinformationen/leitlinie/.

12 Beck RW, Cleary PA. Optic neuritis treatment trial. One-year follow-up results. Arch Ophthalmol. 1993 Jun;111(6):773-5.

13 Beck RW, Cleary PA, Anderson MM Jr, Keltner JL, Shults WT, Kaufman DI, et al.; The Optic Neuritis Study Group. A randomized, controlled trial of corticosteroids in the treatment of acute optic neuritis. N Engl J Med. 1992 Feb;326(9):581-8.

14 Gal RL, Vedula SS, Beck R. Corticosteroids for treating optic neuritis. Cochrane Database Syst Rev. 2015 Aug;(8):CD001430.
15 Kalincik T, Buzzard K, Jokubaitis V, Trojano M, Duquette P, Izquierdo G, et al.; MSBase Study Group. Risk of relapse phenotype recurrence in multiple sclerosis. Mult Scler. 2014 Oct;20(11):1511-22.

16 Lipphardt M, Mühlhausen J, Kitze B, Heigl F, Mauch E, Helms HJ, et al. Immunoadsorption or plasma exchange in steroid-refractory multiple sclerosis and neuromyelitis optica. J Clin Apher. 2019 Aug;34(4):381-91.

17 Heigl F, Hettich R, Arendt R, Durner J, Koehler J, Mauch E. Immunoadsorption in steroid-refractory multiple sclerosis: clinical experience in 60 patients. Atheroscler Suppl. 2013 Jan;14(1):167-73.

18 Ehler J, Koball S, Sauer M, Hickstein H, Mitzner S, Benecke R, et al. Therapeutic plasma exchange in glucocorticosteroid-unresponsive patients with Clinically Isolated Syndrome. Ther Apher Dial. 2014 Oct;18(5):48996.

19 Schilling S, Linker RA, König FB, Koziolek M, Bähr M, Müller GA, et al. [Plasma exchange therapy for steroid-unresponsive multiple sclerosis relapses: clinical experience with 16 patients]. Nervenarzt. 2006 Apr;77(4):430-8.

20 Trebst C, Reising A, Kielstein JT, Hafer C, Stangel M. Plasma exchange therapy in steroid-unresponsive relapses in patients with multiple sclerosis. Blood Purif. 2009;28(2): 108-15.

21 Ehler J, Koball S, Sauer M, Mitzner S, Hickstein $\mathrm{H}$, Benecke R, et al. Response to Therapeutic Plasma Exchange as a Rescue Treatment in Clinically Isolated Syndromes and Acute Worsening of Multiple Sclerosis: A Retrospective Analysis of 90 Patients. PLoS One. 2015 Aug;10(8):e0134583.

22 Hoffmann F, Kraft A, Heigl F, Mauch E, Koehler J, Harms L, et al. Tryptophan immunoadsorption during pregnancy and breastfeeding in patients with acute relapse of multiple sclerosis and neuromyelitis optica. Ther Adv Neurol Disorder. 2018 May;11: 1756286418774973.

23 Stork L, Ellenberger D, Beißbarth T, Friede T, Lucchinetti CF, Brück W, et al. Differences in the Reponses to Apheresis Therapy of $\mathrm{Pa}$ tients With 3 Histopathologically Classified Immunopathological Patterns of Multiple Sclerosis. JAMA Neurol. 2018 Apr;75(4):42835

24 Deschamps R, Gueguen A, Parquet N, Saheb S, Driss F, Mesnil M, et al. Plasma exchange response in 34 patients with severe optic neuritis. J Neurol. 2016 May;263(5):883-7.

25 Roesner S, Appel R, Gbadamosi J, Martin R, Heesen C. Treatment of steroid-unresponsive optic neuritis with plasma exchange. Acta Neurol Scand. 2012 Aug;126(2):103-8.
26 Koziolek MJ, Tampe D, Bähr M, Dihazi H, Jung K, Fitzner D, et al. Immunoadsorption therapy in patients with multiple sclerosis with steroid-refractory optical neuritis. J Neuroinflammation. 2012 Apr;9(1):80.

27 Faissner S, Nikolayczik J, Chan A, Hellwig K, Gold R, Yoon MS, et al. Plasmapheresis and immunoadsorption in patients with steroid refractory multiple sclerosis relapses. J Neurol. 2016 Jun;263(6):1092-8.

28 Kurtzke JF. Rating neurologic impairment in multiple sclerosis: an expanded disability status scale (EDSS). Neurology. 1983 Nov; 33(11):1444-52.

29 Padmanabhan A, Connelly-Smith L, Aqui N, Balogun RA, Klingel R, Meyer E, et al. Guidelines on the Use of Therapeutic Apheresis in Clinical Practice - Evidence-Based Approach from the Writing Committee of the American Society for Apheresis: The Eighth Special Issue. J Clin Apher. 2019 Jun;34(3):171-354.

30 Zantek ND, Boral LI, Li Y, Yamada C, Svensson AM, Crane JE, et al. Hemostasis management and therapeutic plasma exchange: results of a practice survey. J Clin Apher. 2018 Oct;33(5):604-10.

31 Bennett JL, de Seze J, Lana-Peixoto M, Palace J, Waldman A, Schippling S, et al.; GJCFICC\&BR. Neuromyelitis optica and multiple sclerosis: seeing differences through optical coherence tomography. Mult Scler. 2015 May;21(6):678-88.

32 Shen T, You Y, Arunachalam S, Fontes A, Liu S, Gupta V, et al. Differing Structural and Functional Patterns of Optic Nerve Damage in Multiple Sclerosis and Neuromyelitis Optica Spectrum Disorder. Ophthalmology. 2019 Mar;126(3):445-53.

33 Srikajon J, Siritho S, Ngamsombat C, Prayoonwiwat N, Chirapapaisan N; Siriraj Neuroimmunology Research Group. Differences in clinical features between optic neuritis in neuromyelitis optica spectrum disorders and in multiple sclerosis. Mult Scler J Exp Transl Clin. 2018 Aug;4(3): 2055217318791196

34 Keegan M, Pineda AA, McClelland RL, Darby $\mathrm{CH}$, Rodriguez M, Weinshenker BG. Plasma exchange for severe attacks of CNS demyelination: predictors of response. Neurology. 2002 Jan;58(1):143-6.

35 Kleiter I, Gahlen A, Borisow N, Fischer K, Wernecke KD, Hellwig K, et al.; NEMOS (Neuromyelitis Optica Study Group). Apheresis therapies for NMOSD attacks: A retrospective study of 207 therapeutic interventions. Neurol Neuroimmunol Neuroinflamm. 2018 Sep;5(6):e504 